\title{
Tuneable Optical Frequency Comb Generator for THz Spectroscopy
}

\author{
Lalitha Ponnampalam, Martyn J. Fice, Haymen Shams, Cyril C. Renaud and Alwyn J. Seeds \\ Department of Electronic and Electrical Engineering, University College London, WC1E 7JE, UK
}

\begin{abstract}
We present an optical frequency comb generator with a record span of $3.8 \mathrm{THz}$ suitable for high resolution $\mathrm{THz}$ spectroscopy, based on a single phase modulator system. The line spacing, exactly referenced to a microwave synthesizer, can be continuously tuned from $17.5 \mathrm{GHz}$ to $20 \mathrm{GHz}$ overcoming the limitation otherwise imposed by the free spectral range of the fibre loop, making the system capable of generating a continuously tuneable electrical signal from $122.5 \mathrm{GHz}$ to $>2.7 \mathrm{THz}$. The system noise has been measured up to a record frequency of $300 \mathrm{GHz}$, and shown to be limited by the multiplied phase noise of the reference synthesizer.
\end{abstract}

\section{INTRODUCTION}

$\mathrm{D}$ EVELOPMENT of continuously tuneable high spectral purity $\mathrm{THz}$ sources is key to the advancement of $\mathrm{THz}$ spectroscopy so that molecular structures with closely spaced absorption lines can be identified. In order to generate a high spectral purity $\mathrm{THz}$ signal, two suitably spaced comb lines from an optical frequency comb generator (OFCG) can be selected using optical filters and the filtered signal applied to a high bandwidth photomixer, such as an UTC photodiode [1].

In this paper, we report an OFCG with comb lines spanning over $3.8 \mathrm{THz}$, the widest span yet reported from a single phase modulator system without the use of any non-linear mechanisms for spectral extension. Using a variable optical delay line, the line spacing, exactly determined by a microwave reference, can be continuously tuned from $17.5 \mathrm{GHz}$ to $20 \mathrm{GHz}$, limited by the electronic loop components. We also report detailed composite (amplitude + phase) noise measurements of the heterodyne signal up to a record frequency of $300 \mathrm{GHz}$, with an improvement in Optical Signal to Noise Ratio (OSNR) to 50 $\mathrm{dB}$ with the use of injection locked filters.

\section{RESUltS}

The OFCG presented here is based on a single phase modulator within an amplified re-circulating loop using the approach described in $[2,3]$. It consists of a $\mathrm{LiNbO}_{3}$ phase modulator with a $3 \mathrm{~dB}$ bandwidth of $22 \mathrm{GHz}$, a $5 \mathrm{~m}$ long type M12 MetroGain Erbium doped fibre, and a $980 \mathrm{~nm}$ pump laser. The total fibre loop length is $\sim 20 \mathrm{~m}$, giving a free spectral range (FSR) of $\sim 9.3 \mathrm{MHz}$. For optimum operation, the frequency of the microwave reference must be a harmonic of the cavity mode spacing, and the operating frequency of the seed laser must be resonant with one of the cavity modes.

Fig. 1 shows the optical spectrum of the OFCG, with comb lines over a span of $3.8 \mathrm{THz}$, with a $-6 \mathrm{~dB}$ bandwidth of 2.7 THz. The linewidth of each of the comb lines is of the order of $10 \mathrm{~s}$ of $\mathrm{kHz}$, similar to that of the seed laser, and the FWHM linewidth of the electrical spectrum of the heterodyne signal between two adjacent comb lines was measured to be $<10 \mathrm{~Hz}$ (Resolution Bandwidth limited).

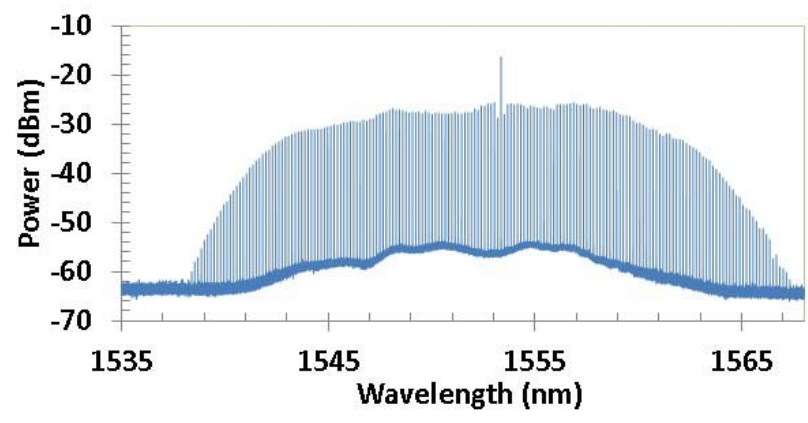

Fig. 1. Optical spectrum of the OFCG (optical resolution $0.04 \mathrm{pm}$ ).

To achieve continuous tuning of the heterodyne signal in the electrical domain, it is necessary to adjust the delay only over the FSR of 9.3 MHz. However, continuous tuning of the comb line spacing of $105 \mathrm{MHz}$ was achieved with a 600 ps optical delay line, when operated on a particular cavity mode as shown in Fig. 2. By tuning to adjacent cavity modes, continuous tuning of the line spacing from $17.5 \mathrm{GHz}$ to $20 \mathrm{GHz}$ (limited by the loop electronics) was realized. This enables continuous heterodyne frequency coverage over the range $122.5 \mathrm{GHz}$ to $>2.7 \mathrm{GHz}$, with additional gapped bands down to $17.5 \mathrm{GHz}$.

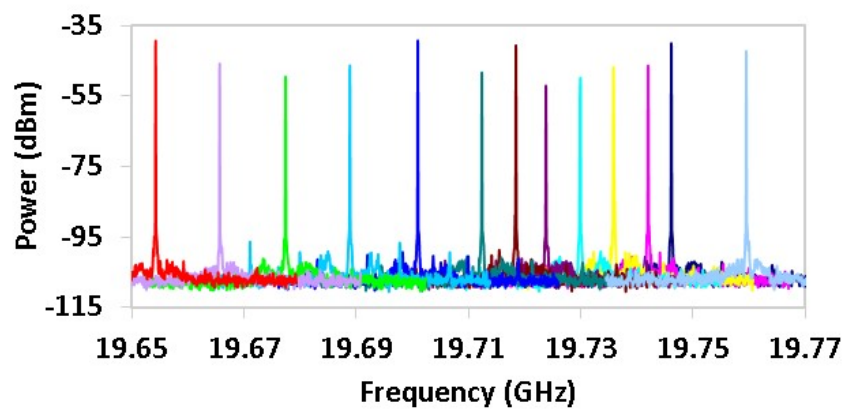

Fig. 2. Tuning of comb line spacing with optical delay line (RBW $1 \mathrm{kHz}$ ).

In this work, the two comb lines separated by the required frequency are filtered by using either a Finisar 4000S waveshaper or by two high power injection locked digital supermode Bragg reflector (DSDBR) lasers from Oclaro Technology, UK. When using the injection locked lasers, an improvement in OSNR to $50 \mathrm{~dB}$ in $0.1 \mathrm{~nm}$ bandwidth is seen, compared to about $25 \mathrm{~dB}$ when a line was selected by the wave shaper.

Fig. 3 shows the schematic of the noise measurement system, where the output of the OFCG is amplified, and the pair of the comb lines are selected using a wave shaper (WS). The pair of suitably spaced comb lines are applied to the high bandwidth UTC photodiode, and the noise of electrical signal at the output of the UTC is analysed using the phase noise function of the Rohde and Schwarz FSQ spectrum analyser. 


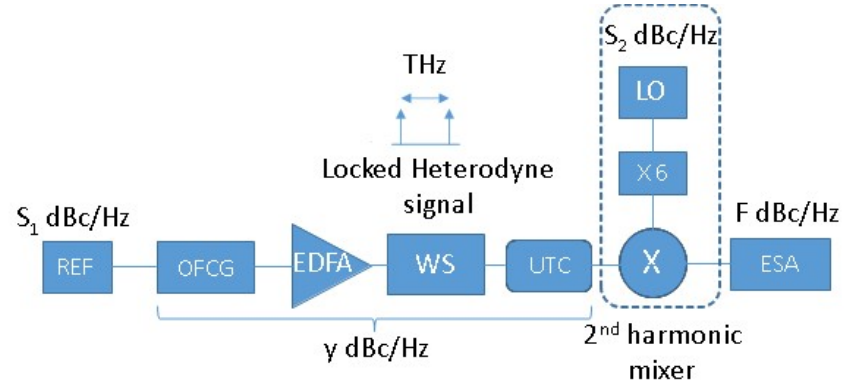

Fig. 3. Schematic of the phase noise measurement arrangement.

However, the measured noise spectral density of the $R \& S$ FSQ spectrum analyser is composed of both amplitude and phase noise. Hence, the measured composite noise of the heterodyne signal between pairs of comb lines separated by multiples of the reference signal frequency up to $296.5 \mathrm{GHz}$ (N $=15$, where $\mathrm{N}$ is the number of comb line spacings between the selected lines) is shown in Fig. 4. The composite noise of two adjacent lines separated by $20 \mathrm{GHz}(\mathrm{N}=1)$ is measured to be less than $-90 \mathrm{dBc} / \mathrm{Hz}$ at an offset frequency of $10 \mathrm{kHz}$, and the noise floors for the measurements shown in Fig. 4 have been calculated to be below the measured noise spectral density [4].

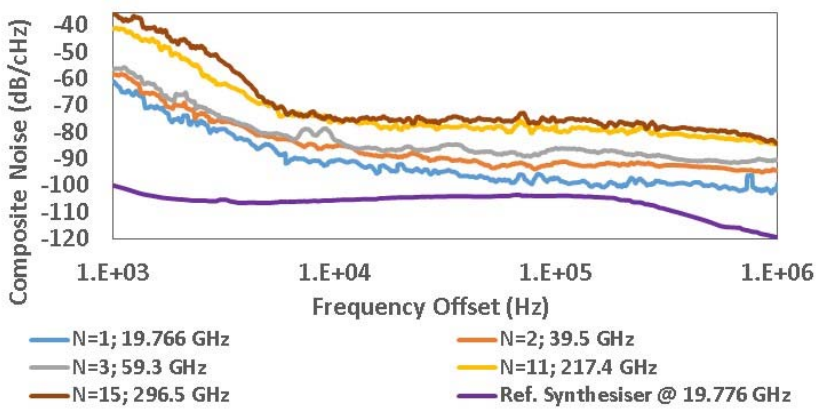

Fig. 4. Composite noise of the beat signal between pairs of comb lines separated by multiples of the comb line spacing.

The overall system noise has been calculated and verified with the measurements shown in Fig. 4. For frequencies less than $40 \mathrm{GHz}$ (ESA bandwidth), the overall system noise has been calculated by combining the phase noise from the reference synthesizer $[\mathrm{S} 1+20 \log (\mathrm{N})] \mathrm{dBc} / \mathrm{Hz}$, contributions from OFCG, EDFA and the UTC photo detector $(\mathrm{y} \mathrm{dBc} / \mathrm{Hz})$ and the $\mathrm{ESA}(\mathrm{F} \mathrm{dBc} / \mathrm{Hz})$. For measurement frequencies greater than $40 \mathrm{GHz}$, where down-conversion was required in the measurement system, scaled phase noise of the local oscillator (LO), $[\mathrm{S} 2+20 \log (12)] \mathrm{dBc} / \mathrm{Hz}$ had been included for the second harmonic mixer with a X6 frequency multiplier (Virginia Diodes WR3.4MixAMC) (Fig. 3). As the measured composite noise at the fundamental frequency is $-96 \mathrm{dBc} / \mathrm{Hz}$ at an offset frequency of $100 \mathrm{kHz}$ (Fig. 4), the combined noise contribution, $\mathrm{y} \mathrm{dBc} / \mathrm{Hz}$ is derived as $-97 \mathrm{dBc} / \mathrm{Hz}$.

The estimated and the measured composite noise of the heterodyne signals at $\mathrm{N}$ times the reference frequency at 100 $\mathrm{kHz}$ offset are shown in Fig. 5 with the scaled phase noise of the reference synthesizer, $[\mathrm{S} 1+20 \log (\mathrm{N})] \mathrm{dBc} / \mathrm{Hz}$. As expected, the composite noise converges with the scaled phase noise of the reference synthesizer at higher multiplication factors, while at lower frequencies $(\mathrm{N} \leq 3)$, the combined contribution, $\mathrm{y} \mathrm{dBc} / \mathrm{Hz}$ is dominant. To achieve a noise level less than $-90 \mathrm{dBc} / \mathrm{Hz}$ at $10 \mathrm{kHz}$ offset at $2 \mathrm{THz}$, it is important to have an ultra-low noise reference synthesizer, with a phase noise less than $-130 \mathrm{dBc} / \mathrm{Hz}$ at $10 \mathrm{kHz}$ offset frequency [5].

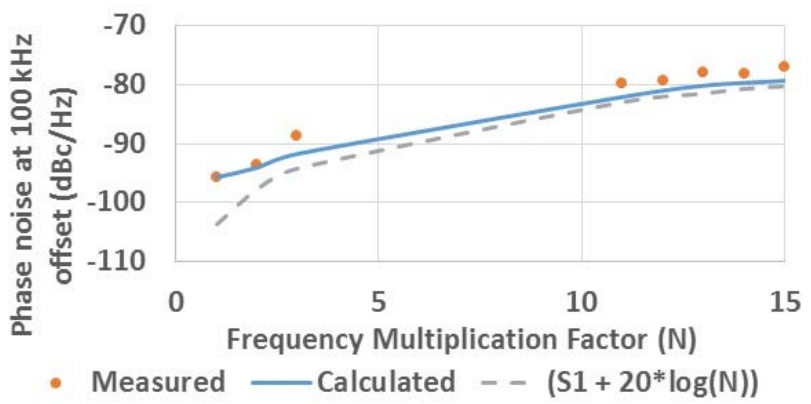

Fig. 5. Measured and calculated noise at $100 \mathrm{kHz}$ offset frequency with the scaled phase noise of the reference synthesizer.

\section{CONCLUSION}

A stable phase coherent optical comb lines over a record span of 3.8 $\mathrm{THz}$ have been demonstrated in a single phase modulator OFCG system. The comb line spacing was continuously tunable from 17.5 $\mathrm{GHz}$ to $20 \mathrm{GHz}$, limited by the loop electronics, and enables the generation of a continuously tunable heterodyne signal from 122.5 $\mathrm{GHz}$ to $>2.7 \mathrm{THz}$ for the first time in such a system. The linewidth of the heterodyne signal between two adjacent comb lines was $<10 \mathrm{~Hz}$ (ESA limited), and the composite noise was less than $-90 \mathrm{dBc} / \mathrm{Hz}$ at an offset frequency of $10 \mathrm{kHz}$. The composite noise was also measured for heterodyne frequencies up to $300 \mathrm{GHz}$ and shown to be limited by multiplied phase noise of the reference source, emphasing the need for an ultra-low noise reference synthesizer for $\mathrm{THz}$ signal synthesis. A tunable $\mathrm{THz}$ signal synthesizer with OSNR greater than $50 \mathrm{~dB}$ has also been realised where two high power widely tunable DSDBR lasers have been injection locked to two of the comb lines separated by the required frequency, while preserving the composite noise characteristics of the OFCG itself. The design described here could be translated to a monolithic integration platform that could lead to compact and portable coherent $\mathrm{THz}$ signal synthesiser, attractive for many applications in the $<3 \mathrm{THz}$ region.

\section{ACKNOWLEDGEMENT}

This work has been supported by the United Kingdom Engineering and Physical Sciences Research Council (EPSRC) through COTS (EP/J017671/1) and HyperTHz (EP/P0218959/1) grants. Authors are grateful to Oclaro Technology, UK for providing widely tunable lasers for optical injection locking.

\section{REFERENCES}

[1]. E. Rouvalis, C. C. Renaud, D. G. Moodie, M. J. Robertson, and A. J. Seeds, Opt. Express, 18, 11105 (2010).

[2]. S. Bennett, B. Cai, E. Burr, O. Gough, and A. J. Seeds, Photon. Technol. Lett., 11, 551 (1999).

[3]. P. Shen, N. J. Gomes, P. A. Davies, P. G. Huggard and B. N. Ellison, J. Lightw. Techn., 25, 3257 (2007).

[4]. L. Ponnampalam, M. J. Fice, H. Shams, C. C. Renaud and A. J. Seeds, Opt. Lett., 43, 2507 (2018).

[5]. T. M. Fortier, M. S. Kirchner, F. Quinlan, J. Taylor, J. C. Bergquist, T. Rosenband, N. Lemke, A. Ludlow, Y. Jiang, C.W. Oates and S. A. Diddams, Nature Photonics, 5, 425 (2011). 